\title{
PREFERENCIA MANUAL EN EL ESPECTRO ESQUIZOFRÉNICO
}

\author{
GISELA LLEBARIA, EVA PRATS, JORDI VICENS-VILANOVA y JORDI E. OBIOLS
}

Universidad Autónoma de Barcelona

(Aceptado en diciembre de 2002)

\begin{abstract}
En la actualidad, la principal hipótesis explicativa de la etiología de la esquizofrenia es la teoría del neurodesarrollo, que postula la existencia de una alteración precoz en el desarrollo del sistema nervioso central (SNC), como base etiológica. Esta alteración hace que el SNC no sea capaz de responder de forma adecuada a las demandas del medio y produce, entre otras, anomalías en el correcto establecimiento de la lateralización cerebral que pueden ser observadas mediante el estudio de la dominancia manual. Por otra parte, diversos estudios muestran que los sujetos que puntúan alto en las subescalas de sintomatologia esquizotipica positiva y desorganizada presentan, con mayor frecuencia, una preferencia manual atípica. El objetivo de este trabajo es el de revisar la condición de preferencia manual atípica como marcador de riesgo en los trastornos del espectro esquizofrénico.
\end{abstract}

Palabras Clave: Preferencia manual atípica, preferencia manual mixta, esquizofrenia, esquizotipia, lateralidad.

\section{Manual preference in the schizophrenic spectrum}

At present, the main framework of schizophrenia etiology is the neurodevelopmental hypothesis that postulates the existence of an abnormal CNS development predating the appearance of the clinical syndrome. This abnormality makes the CNS unable to respond accurately to environmental stress, and implicates anomalies in cerebral lateralization. These anomalies can be observed through manual preference observations. On the other hand, several studies show that the subjects that score high in the positive and disorganized schizotypal symptomatology present, more frequently, an atypical handedness. The purpose of this review article is to check the condition of atypical handedness as a risk marker of the schizophrenic spectrum disorders.

Key words: Atypical handedness, mixed-handedness, schizophrenia, schizotypy, laterality.

\section{PREFERENCIA MANUAL ATÍPICA Y LATERALIDAD EN ESQUIZOFRENIA}

La etiología de los trastornos del espectro esquizofrénico es aún desconocida, pero se cree que podría explicarse por factores genéticos, ambientales o por una combinación de ambos, dando lugar a alteraciones en el neurodesarrollo. Recientes investigaciones se han centrado en el estudio de estas alteraciones, bus-

Correspondencia: Unidad de Investigación en Psicopatología y Neuropsicología, Departamento de Psicología de la Salud y Psicología Social, Facultad de Psicología, Edificio B, Universidad Autónoma de Barcelona, Bellaterra, 08193 Barcelona.

Correo-e: giselallebaria@formabinari.com cando posibles marcadores que permitan detectar anomalías en el proceso de maduración normal del cerebro (Weinberger, 1994).

Estos marcadores suelen ser características evaluables en adultos que reflejan procesos anómalos en el neurodesarrollo que ocurren antes o poco después del nacimiento. Entre estos marcadores se incluyen los dermatoglifos palmares y las alteraciones de la dominancia manual. El posible marcador que centra nuestra atención es la dominancia manual atípica («atypical handedness») como indicador de una lateralización cerebral anómala. 
La lateralidad o asimetría cerebral se deriva de la diferenciación anatómica de los dos hemisferios. Esta diferenciación hemisférica implica una especialización funcional. Existe una base biológica que determina esta especialización hemisférica en la que el hemisferio izquierdo juega un papel principal en todas las funciones del lenguaje y el hemisferio derecho en funciones no verbales y visoespaciales (Molfese y Segalowitz, 1988). En los estudios realizados en población normal, se ha observado que la dominancia manual es, en la mayoría de los casos, contralateral al hemisferio dominante para el lenguaje.

Si el desarrollo normal de la maduración cerebral se ve entorpecido - tanto si es por causas exógenas como genéticasalterará la migración neuronal que se produce durante el segundo trimestre de gestación. Una interferencia en la migración puede producir una alteración en la especialización de funciones.

Estudios con pacientes esquizofrénicos han obtenido pruebas de la existencia de alteración en diferentes lugares del cerebro. Las anomalías se observan comúnmente en el lóbulo temporal medial (Bogerts et al., 1993), en los lóbulos frontales (Klausner, Sweeney, Deck, Haas y Kelly, 1992; Raine et al., 1992) y en la parte medial del diencéfalo (Bornstein, Schwarzkopf, Olson, y Nasrallah, 1992; D'Amato et al., 1992). Los resultados de estas investigaciones concuerdan con las alteraciones en las funciones motoras y en las áreas del lenguaje encontradas en la esquizofrenia. Una alteración en la especialización de las funciones motoras puede ir asociada a una alteración en la lateralización de la preferencia manual. Una alteración que significa un cambio, que puede ser completo hacia la izquierda o incompleto, dando lugar a una ambilateralidad, que se traduciría en una preferencia manual atípica.

\section{¿QUÉ ENTENDEMOS POR PREFERENCIA MANUAL ATÍPICA?}

La preferencia manual atípica se da al producirse un cambio incompleto a la izquierda en la dominancia manual. Esto se traducirá en la no especialización de un solo hemisferio en esta función e implicará la ausencia de una mano dominante. Cabe remarcar que la preferencia manual atípica no radica en la diferenciación entre zurdos y diestros, sino en la distinción entre patrones de lateralidad establecidos y no establecidos, resultando en este último caso una ambilateralidad.

Existen tres tipos de preferencia manual: la preferencia manual diestra, la preferencia manual zurda y la preferencia manual atípica. En lo que se refiere a la preferencia manual atípica, podemos encontrar diferentes subtipos: preferencia manual zurda patológica (pathological left-handedness, PLH), preferencia manual diestra patológica (pathological right-handedness, $P R H$ ), preferencia manual mixta (mixed-handedness, $M H$ ) y preferencia manual ambigua (ambiguous-handedness, $A H$ ). Cabe destacar que el término castellano de ambidextralidad corresponde al término inglés "ambiguous handedness». En la Tabla 1 se resumen estos subtipos.

Entendemos por PLH, la preferencia manual zurda como consecuencia de una lesión precoz en el hemisferio izquierdo. Así se produce un cambio en la preferencia manual natural del sujeto. Del mismo modo, encontramos $\mathrm{PRH}$, es decir, zurdos naturales que debido a una lesión cerebral en el hemisferio derecho cambian su preferencia manual hacia el lado derecho. Presentan $\mathrm{MH}$ aquellas personas que tienen una preferencia manual variable y consistente, mientras que los sujetos con AH utilizan indistintamente cualquiera de las dos manos para una misma tarea. Al pasar el Cuestionario de Preferencia Manual de Annett (Annett, 1970), obser- 
Tabla 1. Subtipos de preferencia manual atípica

\begin{tabular}{cc}
$\begin{array}{c}\text { PLH } \\
\begin{array}{c}\text { Pathological left-handedness } \\
\text { Preferencia manual zurda patológica }\end{array}\end{array}$ & $\begin{array}{c}\text { Sujetos cuya preferencia manual es zurda como consecuencia de } \\
\text { una lesión precoz en el hemisferio izquierdo }\end{array}$ \\
\hline $\begin{array}{c}\text { PRH } \\
\text { Preferencia manual diestra patológica }\end{array}$ & $\begin{array}{c}\text { Sujetos cuya preferencia manual es diestra como consecuencia de } \\
\text { una lesión precoz en el hemisferio derecho }\end{array}$ \\
\hline MH & Sujetos cuya preferencia manual es variable y consistente \\
$\begin{array}{c}\text { Mixed-handedness } \\
\text { Preferencia manual mixta }\end{array}$ & \\
Ambiguous-handedness & Sujetos cuya preferencia manual es variable e inconsistente \\
Preferencia manual ambigua &
\end{tabular}

varíamos que el sujeto $\mathrm{MH}$ utiliza, por ejemplo, siempre la mano derecha para escribir, coger una raqueta y lavarse los dientes, y siempre la mano izquierda para repartir cartas, abrir un frasco y clavar un clavo. En cambio, en el caso de un sujeto $\mathrm{AH}$ encontraríamos que utiliza indistintamente la mano derecha $e$ izquierda para escribir, coger una raqueta o lavarse los dientes, por ejemplo (Satz y Green, 1999).

Estos dos subtipos de preferencia manual atípica se dan como consecuencia de un patrón de lateralización no completo (ambilateralidad).

\section{RELACIÓN ENTRE MH Y ESQUIZOFRENIA}

En los primeros estudios se consideró que entre la población esquizofrénica existía una mayor prevalencia de zurdos que en la población general. Actualmente, se sabe que la mayor prevalencia no es de zurdos sino de no-diestros, incluyendo en este grupo tanto a los zurdos como a los que presentan una preferencia manual mixta (MH) y ambigua (AH).

$\mathrm{El}$ hecho de que en los primeros estudios se pensara en un aumento de la prevalencia de preferencia manual zurda dentro de la población esquizofrénica se debe a ciertas irregularidades metodológicas. Las dos irregularidades principales eran considerar la preferencia manual de forma dicotómica (derecha-izquierda) y evaluarla con un solo ítem (preferencia manual a la hora de escribir). El cambio de metodología en dicha evaluación permitió descubrir que la mayor prevalencia era de $\mathrm{MH}$ y no de zurdos.

Esta hipótesis queda avalada por los experimentos realizados por Green, Satz, Smith y Nelson (1989) y por Nelson, Satz, Green y Cicchietti (1993). En ellos se obtuvo un porcentaje de $\mathrm{MH}$ del $40 \%$ en pacientes esquizofrénicos frente a un $15 \%$ en el grupo control. Cabe tener en cuenta, que la mitad de los MH cumplían los criterios para ser considerados $\mathrm{AH}$. Estos estudios tienen como antecedentes los de Walker y Birch (1970); Dvirskii (1976); Boklage (1977); Gur (1977); Nasrallah, Keelor, Van Schroeder y McCallyWhitters (1981); Shan-Ming et al., (1985).

Estudios más recientes muestran, en general, la misma tendencia. Así las investigaciones realizadas por Clementz, Iacono y Beiser (1994), Manoach (1994) o Cannon et al, (1995), corroboran la hipótesis de que en la población esquizofrénica se da un cambio hacia la izquierda en la preferencia manual como resultas de una anormalidad sucedida en períodos tempranos de la maduración neuronal. 


\section{PREFERENCIA MANUAL ATÍPICA Y SINTOMATOLOGÍA CLÍNICA}

Un establecimiento anómalo de la lateralidad no sólo implica una dominancia manual atípica, sino que también predice la presencia de alteraciones comportamentales, cognitivas, neurológicas y anatómicas asociadas a la esquizofrenia. Posteriores estudios centraron su atención en determinar si existía relación entre la dominancia manual atípica y otros síntomas presentes en esquizofrenia.

Taylor, Dalton y Fleminger (1982) estudiaron la relación entre preferencia manual y sintomatología psiquiátrica en 232 pacientes esquizofrénicos. Los investigadores dividieron el grupo experimental según presentaran mayor número de síntomas positivos o negativos. Compararon las distribuciones de preferencia manual dentro de cada subgrupo. Sólo el trastorno formal de pensamiento fue relacionado con los sujetos con LH y MH.

Posteriormente, Manoach, Maher y Manschreck (1988) evaluaron la preferencia manual y el trastorno formal del pensamiento en 58 varones esquizofrénicos. Encontraron una mayor prevalencia de trastorno formal del pensamiento en sujetos con LH. Cabe destacar que la evaluación de la dominancia manual únicamente se determinó mediante la mano utilizada para escribir. Probablemente, una evaluación más exhaustiva hubiera precisado que muchos de los etiquetados como LH eran MH.

Manoach (1994) replicó el estudio realizado en 1988. En esta ocasión utilizó un grupo de pacientes esquizofrénicos, un grupo de pacientes psiquiátricos no esquizofrénicos y un grupo control normal. La evaluación de la dominancia manual se realizó de forma dimensional. Los resultados fueron similares a los obtenidos en el anterior estudio, es decir, encontró en los pacientes esquizofrénicos una relación entre LH o MH y un aumento de los trastornos formales del pensamiento y de las disfunciones del lenguaje en pacientes esquizofrénicos, pero no los halló en el grupo control de pacientes psiquiátricos ni en el grupo de controles normales.

El estudio realizado por Tyler, Diamond y Lewis (1995) también mostró una mayor prevalencia de alucinaciones auditivas en pacientes esquizofrénicos zurdos. La muestra constaba de 94 zurdos y 595 diestros, cuya evaluación de la preferencia manual se hizo mediante una sola variable. Este estudio también encontró que los pacientes zurdos tenían mayor número de alteraciones cognitivas y comportamentales en la infancia que los pacientes diestros.

Manschreck, Maher, Redmon, Miller y Beaudette (1996) investigaron la relación entre LH, memoria y trastorno formal del pensamiento en esquizofrenia. La evaluación de la preferencia manual se realizó mediante un único ítem de la escala de Annett, resultando la población del estudio 21 pacientes diestros y 21 pacientes zurdos. Los resultados reflejaron que los pacientes zurdos tenían mayor número de síntomas del trastorno formal de pensamiento (descarrilamiento, ilogicalidad, etc.).

Los resultados que obtuvieron todos estos estudios avalan la correlación citada y hacen pensar en una misma causa tanto para el cambio en la dominancia manual como para la reducción de especialización en las áreas relacionadas con el lenguaje.

Taylor y Amir (1995) tomaron tres grupos: uno de pacientes esquizofrénicos, otro de pacientes con trastornos afectivos y otro grupo de controles normales, que fueron evaluados con la batería Oldfield. Estos autores no encontraron ninguna relación entre zurdos o $\mathrm{MH}$ y trastorno. formal del pensamiento, manía, depresión o embotamiento emocional. Tampo- 
co encontraron un aumento en la proporción de no-diestros dentro del grupo de pacientes esquizofrénicos.

\section{PREFERENCIA MANUAL ATÍPICA Y ESQUIZOTIPIA}

Hay una creciente tendencia a considerar la esquizofrenia dentro de un continuum que va desde la normalidad hasta los trastornos psicóticos y no como una entidad categórica. El espectro esquizofrénico es el rango que parte desde la personalidad inadecuada (personalidad esquizotípica) hasta la esquizofrenia y se basa en la existencia de un grupo de estados psicopatológicos que comparten una etiología común con la esquizofrenia. Por tanto, este continuum sería una gradación en cuanto a gravedad y número de síntomas psicóticos en función de la presencia de un mayor o menor número de factores de riesgo.

Basándonos en la teoría del continuum es razonable pensar que podemos encontrar también una mayor proporción de dominancia manual atípica en individuos normales con rasgos propios de la personalidad esquizotípica. Esta hipótesis fue estudiada por Chapman y Chapman (1987) en una muestra de 7457 estudiantes. Se evaluó la preferencia manual y rasgos de personalidad esquizotípica mediante la Escala de Percepción Aberrante - Ideación mágica («Perceptual Aberration - Magical Ideation Scale». Chapman, Chapman y Raulin, 1978; Eckblad y Chapman, 1983), Escala de Impulsividad no conformista («Impulsive Nonconformity Scale». Chapman, Chapman, Numbers, Edell, Carpenter y Beckfield, 1984) y la Escala de Anhedonia Física ( Physical Anhedonia Scale». Chapman, Chapman y Raulin, 1976).

Los resultados apoyaron esta hipótesis, pues se encontró una alta preferencia manual no diestra en aquellos indi- viduos que puntuaron alto en las escalas de Percepción Aberrante y de Ideación Mágica. Similares hallazgos obtuvieron Kim, Raine, Triphon y Green (1992) utilizando el Cuestionario de Personalidad Esquizotípica ( Schizotypal Personality Questionnaire», SPQ. Raine, 1991). Encontraron una correlación entre la preferencia manual atípica y aquellos rasgos esquizotípicos asociados a una disfunción cognitivo-perceptiva. Concretamente, se halló una relación entre $\mathrm{MH}$ y las subeşcalas «habla extravagante», "comportamiento excéntrico», "suspicacia» $\mathrm{y}$ «experiencias perceptivas no habituales».

En la misma línea, Barrantes-Vidal, Vicens-Vilanova, Subirà y Obiols (2002a,b) hallaron relación entre $\mathrm{MH}$ y las dimensiones positivas y desorganizadas de la esquizotipia.

De forma paralela a la relación existente entre esquizofrenia y preferencia manual atípica, en estos estudios se halla una correlación entre sintomatología positiva y $\mathrm{MH}$.

Un estudio reciente es el de Shaw, Claridge y Clark (2001), realizado con una muestra de 3.450 universitarios que respondieron a un cuestionario enviado por correo electrónico. Los rasgos de personalidad esquizotípica fueron evaluados mediante el Inventario OxfordLiverpool de Sentimientos y Experiencias («Oxford-Liverpool Inventory of Feelings and Experiences", OLIFE. Mason, Claridge y Jackson, 1995) y la preferencia manual atípica mediante el Cuestionario de Preferencia Manual de Annett (Annett, 1970). En este estudio se halló una correlación entre la sintomatología positiva de la esquizotipia (determinada por la subescala «Experiencias inusuales» del OLIFE) y preferencia manual atípica. A diferencia de estudios anteriores, esta relación no sólo se ha encontrado con $\mathrm{MH}$, sino también con $\mathrm{AH}$. 


\section{LA IMPORTANCIA DEL TIPO DE TAREA}

Poreh (1994) pudo respaldar la relación entre esquizotipia y preferencia manual atípica, pero no con la misma significación. Según este autor, estas diferencias de resultados podrían ser explicadas por el hecho de que en los diferentes estudios las medidas de preferencia manual no eran las mismas y apuntó la necesidad de diferenciar entre el tipo de tareas según requieran más o menos habilidad.

El aprendizaje de una tarea motora que requiere una cierta habilidad es lento ya que la coordinación de los diferentes grupos musculares es escasa. Dicho aprendizaje precisa de un elevado número de ensayos hasta su correcta ejecución. En este momento la acción ya está aprendida y la secuencia de movimientos coordinados automatizada. Esto hace pensar que cuanto más compleja es la acción a aprender, más ventajoso es lateralizar el aprendizaje. Por lo tanto, cabe esperar una mayor lateralización de la preferencia manual en tareas que requieran mayor habilidad.

La relación entre el tipo de tarea y la preferencia manual en esquizofrenia fue estudiada por Nelson et al. (1993) y hallaron que para las tareas en las que se requería menos habilidad estos sujetos tendían a mostrar $\mathrm{MH}$, pero que eso no ocurría cuando la tarea requería más habilidad. Estos resultados apuntan hacia una posible inestabilidad en la preferencia manual en la esquizofrenia que podría ser susceptible a factores atencionales.

Extrapolando estos resultados a la personalidad esquizotípica, Poreh, Levin, Teves y States (1997) estudiaron la relación entre ésta y la preferencia manual según el tipo de tarea (según requieran mayor/menor habilidad) en una muestra de 308 estudiantes de una media de edad de 21,3 años. Las medidas que se utilizaron para determinar la lateralidad fueron el Inventario de Preferencia Manual de
Oldfield ( Oldfield Handedness Inventory», OHI. Oldfield, 1971) y el Cuestionario de Preferencia Manual de Waterloo ( "Waterloo Handedness Questionnaire», WHQ. Steenhuis y Bryden, 1989). El OHI evalúa tareas habilidosas mientras que la WHQ diferencia las tareas que requieren mayor habilidad de las que requieren menos habilidad. Para evaluar los rasgos de personalidad esquizotípica se utilizó el SPQ.

Los resultados corroboran, en parte, los obtenidos anteriormente por Kim et al. (1992). En ambos casos se confirma la mayor proporción de $\mathrm{LH}$ y $\mathrm{MH}$ en la población normal con rasgos esquizotípicos. También se encontró una correlación significativa entre una preferencia para tareas que requieran habilidad y algunos rasgos esquizotípicos asociados con déficits cognitivo-perceptivos. También se halló correlación entre altas puntuaciones en el SPQ y preferencia manual no diestra para tareas que implican habilidad (al contrario de lo encontrado en pacientes esquizofrénicos). Por último, resaltar el hecho de que algunos individuos con rasgos esquizotípicos no mantienen su preferencia manual no diestra, corroborando la hipótesis de que variables situacionales influyen en la dominancia manual en esta población. Estos resultados apuntan en la misma dirección que los de Nelson et al (1993).

\section{DISLEXIA, ESQUIZOTIPIA Y PREFERENCIA MANUAL ATÍPICA}

Las razones por la que se ha querido estudiar la relación entre esquizotipia y dislexia son, por una parte, la existencia de una alta prevalencia de no diestros entre los disléxicos y sus familiares, y por otra, el hecho de que la clave de la dislexia se encuentre en una lateralización cerebral anómala (Molfese y Segalowitz, 1988; Geschwind y Galaburda, 1987). 
Richardson y Stein (1993) investigaron las características de personalidad de un grupo de disléxicos y un grupo control mediante el Cuestionario de Personalidad de Eysenck ( Eysenck Personality Questionnaire», EPQ; Eysenck y Eysenck, 1975) y las Escalas de Personalidad Esquizotípica y Límite ( Schizotypal and Borderline Personality», STA y STB; Claridge y Broks, 1984). Se encontraron altas puntuaciones en la STA en el grupo de disléxicos. Un análisis más detallado de estos datos apunta una tendencia a las experiencias cognitivas y perceptivas no habituales en el grupo de disléxicos, es decir, a la sintomatología positiva.

También ha sido estudiada la preferencia manual en la dislexia, encontrándose una alta prevalencia de $\mathrm{MH}$ entre la población disléxica (Annett y Kilshaw, 1984).

La relación entre dislexia, preferencia manual atípica y rasgos esquizotípicos fue estudiada de forma más exhaustiva por Richardson (1994). El estudio se realizó con una muestra de 50 disléxicos -27 hombres y 23 mujeres- y un grupo control de 82 sujetos. Richardson se basó en dos modelos teóricos para evaluar la esquizotipia. El primero de estos modelos es el Modelo de Tres Síndromes de la Esquizotípia (Gruzelier, 1994 a,b; Gruzelier, Burgess, Stygall, Irvin y Raine, 1994) y comprende los factores "Actividad" («Active»), «Introversión» («Withdrawn») e «Irrealidad» («Unreality»). El segundo modelo tiene cuatro factores que son: «Experiencias inusuales» («Unusual experiences»), "Anhedonia Introvertida» («Introvertive Anhedonia»), "Impulsividad no conformista" ( Impulsive Nonconformity") y "Desorganización Cognitiva» ("Cognitive Disorganization»). De este último modelo surgió el Inventario Oxford-Liverpool de Sentimientos y Experiencias ( Oxford-Liverpool Inventory of Feelings and Experiences», OLI-
FE. Mason et al., 1995), para evaluar la propensión a la psicosis.

Los resultados mostraron altas puntuaciones del grupo disléxico en los síntomas positivos del modelo de tres factores ("Actividad» e «Irrealidad»). En el modelo de los cuatro factores sólo se encontraron altas puntuaciones del grupo de disléxicos en el factor «Experiencias Inusuales".

Estos resultados muestran una asociación entre dislexia y sintomatología positiva. Esto concuerda con el trabajo de Chapman y Chapman (1987) y sugieren que esquizotipia y dislexia puedan compartir bases biológicas, probablemente una lateralización cerebral anómala. Esta hipótesis es avalada por la alta prevalencia de disléxicos en los familiares de esquizofrénicos. Además, esquizofrenia y dislexia también comparten otros rasgos como problemas atencionales, déficit en el procesamiento visual, anormalidades en el seguimiento ocular y pobre coordinación motora.

En cuanto a preferencia manual, se halló una mayor prevalencia de $\mathrm{MH}$ en el grupo de disléxicos, resultado que reafirma la hipótesis de una anómala lateralización en la dislexia. También se encontró una alta prevalencia de $\mathrm{MH}$ en aquellos individuos del grupo control que mostraron una propensión a las psicosis y en aquellas medidas que se referían a distorsiones perceptivas.

\section{CONCLUSIONES}

En los primeros estudios realizados sobre preferencia manual en la esquizofrenia, se observó una mayor prevalencia de zurdos que en la población normal. Un cambio de metodología - pasó de ser dicotómica y con un solo ítem, a ser más exhaustiva- mostró que la mayor prevalencia era de sujetos con preferencia manual mixta $(\mathrm{MH})$ y no de zurdos, 
demostrándose así la existencia de una relación entre preferencia manual atípica y esquizofrenia.

Posteriormente, el interés se centró en estudiar la relación entre sintomatología psicótica y preferencia manual atípica. En este tipo de estudios, sólo se estableció relación entre la sintomatología positiva (alucinaciones), la desorganizada (trastorno formal del pensamiento) y la población esquizofrénica no diestra. Una cuestión esencial en la que cabría profundizar es el motivo por el cuál la preferencia manual atípica sólo se ha podido relacionar con este tipo de sintomatología y no con la sintomatología negativa.

También ha sido estudiada la relación entre preferencia manual atípica y rasgos esquizotípicos de la personalidad en población normal. En estos trabajos se observa la misma tendencia que en los realizados con muestras clínicas de pacientes esquizofrénicos, si bien, en este caso, los resultados son más concluyentes hacia una relación entre sujetos $\mathrm{MH}$ y este tipo de rasgos.

Los trastornos del espectro esquizofrénico y la dislexia comparten características como: déficit atencional, pobre coordinación motora, anormalidades en el seguimiento ocular, etc. Además, existe una mayor prevalencia tantó de sujetos MH como de sujetos con rasgos de personalidad esquizotípica en la población disléxica respecto a población general. Todo ello, añadido al hecho de que en la dislexia se da una lateralización anómala, hace pensar que en ambos trastornos podría existir una etiología común.

En algunos de los estudios revisados existen problemas metodológicos en la evaluación de la preferencia manual, por tanto sería apropiado que en futuros estudios dicha evaluación se estandarizara. En este sentido, el Cuestionario de Preferencia Manual de Annett (Annett, 1970) podría ser un instrumento adecuado, registrando además aquellos items en los que el sujeto carezca de una mano dominante.

Otro aspecto importante a tener en cuenta respecto a la preferencia manual, es la diferenciación entre el tipo de tarea, según requiera mayor o menor habilidad. La importancia de esta variable radica en que la preferencia manual podría ser sensible a factores de tipo atencional, típicamente asociados a las patologías del espectro esquizofrénico.

En conclusión, los resultados obtenidos en los diferentes estudios revisados apoyan la posibilidad de que la preferencia manual atípica sea un marcador de la patología del espectro esquizofrénico. Esta suposición constituye, por una parte, un dato a favor de la teoría del neurodesarrollo y, por otra, implicaría, en caso de confirmarse, un avance en la detección precoz de los trastornos psicóticos de inicio juvenil.

\section{REFERENCIAS BIBLIOGRÁFICAS}

Annett, M. (1970). A classification of hand preference by association analysis. British Journal of Psychology, 67, 587-592.

Annett, M., y Kilshaw, D. (1984). Lateral preference and skill in dyslexics: implications of the right shift theory. Journal of child Psychology and Psychiatry, 25, 357-377.

Barrantes-Vidal, N., Vicens-Vilanova, J., Subirà, S., y Obiols, J.E. (2002a). Association between mixed-handedness and cognitive disorganisation in an adolescent general population sample. Schizophrenia Research, 53, 90.

Barrantes-Vidal, N., Vicens-Vilanova, J., Subirà, S., y Obiols, J.E. (2002b). Handedness and schizotypy in an adolescent community sample. Acta Psychiatrica Scandinavica, 413, Suppl. 79. F.I.: 1.774.

Boklage, C.E. (1977). Schizophrenia, brain asymmetry, development and prognostic implications. Biological Psychiatry, 12, 812-816. 
Bogerts, B., Lieberman, J.A., Ashtari, M., Bilder, R.M., Degreef, G., Johns, C., y Masiar, $S$. (1993). Hippocampus-amygdala volumes and psychopathology in chronic schizophrenia. Biological Psychiatry, 33, 236-246.

Bornstein, R.A., Schwarzkopf, S.B., Olson, S.C., y Nasrallah, H.A. (1992). Third-ventricle enlargement and neuropsychological deficit in schizophrenia. Biological Psychiatry, 31, 954-961.

Cannon, M., Byrne, M., Cassidy, B., Larkin, C., Horgan, R., Sheppard, N.P., y O'Callaghan, E. (1995). Prevalence and correlates of mixed-handedness in schizophrenia. Psychiatry Research, 59, 119-125.

Champan, L.J., Chapman, J.P., Numbers, J.S., Edell, W.S., Carpenter, B.N., y Beckfield, D. (1984). Impulsive nonconformity as a trait contributing to the prediction of psychoticlike and schizotypal symptoms. Journal of Nervous and Mental Disease, 172, 681-691.

Chapman, J.P., y Chapman, L.J. (1987). Handedness of hypothetically psychosis-prone subjects. Journal of Abnormal Psychology, 96, 89-93.

Chapman, J.P., Chapman, L.J., y Raulin, M.L. (1978). Body-image aberration in schizophrenia. Journal of Abnormal Psychology, 87, 399-407.

Chapman, J.P., Chapman, L.J., y Raulin, M.L. (1976). Scales for physical and social anhedonia. Journal of Abnormal Psychology, 85, 374-382.

Claridge, G.S., y Broks, P. (1984). Schizotypy and hemisphere function. I. Theoretical considerations and measurement of schizotypy. Personality and Individual Differences, 5, 633-648.

Clementz, B.A., Iacono, W.G., y Beiser, M. (1994). Handedness in first-episode psychotic patients and their first-degree biological relatives. Journal of Abnormal Psychology, 103, 400-403.

D'Amato, T., Rochet, T., Dalery, J., Laurent, A., Chauchat, J.H., Terra, J.L., y Marie-Cardine, M. (1992). Relationship between symptoms rated with the Positive and Negative Syndrome Scale and brain measures in schizophrenia. Psychiatry Research, 44, 55-62.

Dvirskii, A. E. (1976). Functional asymmetry of the cerebral hemispheres in clinical types of schizophrenia. Nervous and Behavioral Physiology, 7, 236-239.

Eckblad, M., y Chapman, L.J. (1983). Magical ideation as an indicator of schizotypy. Journal of Consulting and Clinical Psychology, 51, 215-225.

Eysenck, H.J., y Eysenck, S.G.B. (1975). Manual of the Eysenck Personality Questionnaire. London: Hodder \& Stonghton. Geschwind, N., y Galaburda, A.M. (1987). Cerebral lateralization: biological mechanisms. Associations and pathology. Cambridge, MA: MIT Press.

Green, M.F., Satz, P., Smith, C., y Nelson, L. (1989). Is there atypical handedness in schizophrenia? Journal of Abnormal Psychology, 98, 57-65.

Gruzelier, J.H. (1994a). Syndromes of schizotypy: Patterns of cerebral laterality, arousal and gender. En A. Raine, T. Lencz y S. Mednick (Eds.), Schizotypal Personality. Cambridge University Press (en prensa).

Gruzelier, J.H. (1994b). Developmental psychopathology: brain asymmetry, gender and maduration. International Journal of Psychophysiology, 18 (en prensa).

Gruzelier, J., Burgess, A., Stygall, J., Irving, G., y Raine, A. (1994). Patterns of cognitive asymmetry and syndromes of schizotypal personality. Psychiatry Research (en prensa).

Gur, R.E. (1977). Motoric laterality imbalance in schizophrenia. Archive of General Psychiatry, 34, 33-37.

Kim, D., Raine, A., Triphon, N., y Green, M.F. (1992). Mixed handedness and features of schizotypal personality in a non-clinical sample. Journal of Nervous and Mental Disease, 180,133-135.

Klausner, J.D., Sweeney, J.A., Deck, M.D.F., Haas, G.L., y Kelly, A.B. (1992). Clinical correlates of cerebral ventricular enlargement in schizophrenia. Further evidence for frontal lobe disease. Journal of Nervous and Mental Disorders, 180, 407-412.

Manoach, D.S. (1994). Handedness is related to formal thought disorder and language dysfunction in schizophrenia. Journal of Clinical and Experimental Neuropsycho$\log y, 16,2-14$.

Manoach, D.S., Maher, B.A., y Manschreck, T.C. (1988). Left-handedness and thought 
disorder in the schizophrenias. Journal of Abnormal Psychology, 97, 97-99.

Manschreck, T.C., Maher, B.A., Redmond, D.A., Miller, C., y Beaudette, S.M. (1996). Laterality, memory, and thought disorder in schizophrenia. Neuropsychiatry, Neuropsychology, and Behavioral Neurology, 9, 1-7.

Mason, O., Claridge, G., y Jackson, M. (1995). New scales for the assessment of schizotypy. Personality and Individual Differences, 18, 7-13.

Molfese, D.L., y Segalowitz, S.J. (1988). Brain Lateralization in Children: Developmental Implications. New York: Guilford Press.

Nasrallah, H.A., Keelor, K., Van Schroeder, C., y McCally-Whitters, M. (1981). Motoric laterality in schizophrenic males. American Journal of Psychiatry, 39, 1006-1010.

Nelson, L.D., Satz, P., Green, M., y Cicchietti, D. (1993). Re-examining handedness in schizophrenia - now you see it - now you don't. Journal of Clinical and Experimental Neuropsychology, 15, 149-158.

Oldfield, R.C. (1971). The assessment of handedness: The Edinburgh Inventory. $\mathrm{Neu}$ ropsychologia, 9, 97-113.

Poreh, A. (1994). Reexamination of mixed handedness in psychosis-prone college students. Personality and Individual Differences, $17,445-448$.

Poreh, A.M., Levin, J., Teves, H., y States, J. (1997). Mixed handedness and schizotypal personality in a non-clinical sample - the role of task demand-. Personality and Individual Differences, 23, 501-507.

Raine, A., Lencz, T., Reynolds, G.P., Harrison, G., Sheard, C., Medley, I., Reynolds, L.M., y Cooper, J.E. (1992). An evaluation of structural and functional prefrontal deficits in schizophrenia: MRI and neuropsychological measures. Psychiatry Research Neuroimaging, 45, 123-137.

Raine, A. (1991). The SPQ: A scale for the assessment of schizotypal personality based on DSM-III criteria. Schizophrenia Bulletin, 17, 555-564.

Richardson, A.J. (1994). Dyslexia, handedness and syndromes of psychosis prone- ness. International Journal of Psychophysiology, 18, 251-263.

Richardson, A.J., y Stein, J.F. (1993). Personality characteristics of adult dyslexics. En S.F. Wright y R. Groner (Eds.), Facets of Dyslexia and its Remediation. Amsterdam: Elsevier/North-Holland Science Publishers (pp. 411-423).

Satz, P., y Green, M.F. (1999). Atypical handedness in Schizophrenia: Some methodological and theoretical issues. Schizophrenia Bulletin, 25, 63-78.

Shan-Ming, Y., Flor Henry, P., Dayi, C., Tiangi, L., Shunguang, Q., y Zenxiang, M. (1985). Imbalance of hemispheric functions in major psychoses: A study of handedness in the People's Republic of China. Biological Psychiatry, 20, 906-917.

Shaw, J., Claridge, G., y Clark, K. (2001). Schizotypy and the shift from dextrality: a study of handedness in a large non-clinical sample. Schizophrenia Research, 50, 181189.

Steenhuis, R.E., y Bryden, M.P. (1989). Different dimensions of hand preference that relate to skilled and unskilled activities. Cortex, 25, 289-304.

Taylor, M.A., y Amir, N. (1995). Left-handedness in schizophrenia and affective disorder. Journal of Nervous and Mental Disease, 183, 3-9.

Taylor, P., Dalton, R., y Fleminger, J.J. (1982). Handedness and schizophrenic symptoms. British Journal of Medical Psychology, 55, 287-291.

Tyler, M., Diamond, J., y Lewis, S. (1995). Correlates of Left-handedness in a large sample of schizophrenic patients. Schizophrenia Research, 18, 37-41.

Walker, H.A., y Birch, H.C. (1970). Lateral preference and right-left awareness in schizophrenic children. Journal of Nervous and Mental Disease, 151, 341-351.

Weinberger, D.R. (1994). Schizophrenia as a neurodevelopmental disorder: a review of the concept. En J.R. Hirsch y D.R. Weiberger (Ed.), Schizophrenia. Londres: Blakwood Press. 